\title{
Trend analysis and computational statistical estimation in a stochastic Rayleigh model: Simulation and application
}

\author{
R. Gutiérrez*, R. Gutiérrez-Sánchez, A. Nafidi \\ Department of Statistics and Operational Research, University of Granada, \\ Facultad de Ciencias, Campus de Fuentenueva, 18071 Granada, Spain
}

Available online 31 August 2007

\begin{abstract}
This paper considers a stochastic model based on the homogeneous stochastic Rayleigh diffusion process. We first examine the main probabilistic characteristics of the model and describe, among other results, an explicit expression of the trends (both conditioned and nonconditioned) and, when it exists, the stationary distribution. We then obtain results of the statistical estimation of the corresponding parameters and consider the computational problems that may arise. In addition, we present an algorithm for the stochastic simulation of the sample path of the model based on the corresponding Ito stochastic differential equation. Finally, the model is applied to study the evolution of the production of thermal electricity in countries in the Maghreb region; the results obtained are in good statistical accord with the real data observed for the period 1980-2002.
\end{abstract}

(C) 2007 IMACS. Published by Elsevier B.V. All rights reserved.

Keywords: Stochastic diffusion process; Rayleigh model; Estimation; Simulation; Electricity production in the Maghreb

1991 MSC: 60J60; 62M05

\section{Introduction}

It is well known that in stochastic modelling an outstanding role is played by stochastic diffusion processes; these are considered either from the viewpoint of the corresponding Ito or Stratonovich stochastic differential equations (SDEs) or from the associated Kolmogorov (Fokker-Planck and backward) differential equations. Stochastic diffusions have been widely used in diverse fields, such as stochastic financial analysis, animal or cell growth in a random environment, marketing, and the natural environment. In particular, Gompertz and lognormal stochastic diffusion processes have been studied with respect to specific theoretical aspects, and they have been successfully applied to real cases in Gutiérrez et al. [12,10] and Ferrante et al. [2]. In order to apply these diffusion processes to the modelling and prediction of real phenomena, it is necessary to develop results of statistical inference, firstly on the estimation of their parameters (general results on this question can be consulted in Prakasa Rao [16]). The corresponding statistical methodology is based on a continuous sampling of the sample paths or on time-discretised observations of the dynamic variable under consideration. This may, however, run into problems related to computation and to numeric approximations with respect to the resolution of nonlinear equations and the calculation of integrals. The algorithms used to simulate the sample paths of the processes considered must also be based upon discretised approximations of the corresponding Ito

\footnotetext{
* Corresponding author. Tel.: +34 958243267; fax: +34 958243267.

E-mail address: rgjaimez@ugr.es (R. Gutiérrez).
} 
stochastic equations. This methodology has been considered by several authors recently; specifically, for Gompertz and logistic lognormal diffusion processes, real results and applications are available, see for example Gutiérrez et al. [11,9] and Giovanis and Skiadas [4]. With respect to the Rayleigh process considered in the present paper, we study the abovementioned questions and establish a methodology that enables it to be statistically fitted to real observed data. In Section 2 we consider basic theoretical results of a probabilistic nature that complement the results obtained by Giorno et al. [3], and we obtain the moment functions expressed in terms of Kummer functions, as well as the stationary distribution. In particular, we obtain the trends (the conditioned and the nonconditioned mean functions). Subsequently, in Section 3 , we present results of the estimation of the parameters of the model, obtained by the continuous sampling of sample paths and by the maximum likelihood method; we then propose a methodology to resolve the computational problems that may arise in its calculation. Finally, in Section 4 we analyse the modelling procedure, using the Rayleigh model proposed, of the dynamic evolution of the trend corresponding to thermal electricity production in countries within the Maghreb region, using real data for annual production for the period 1980-2002. The variable that is considered is of great interest in environmental scientific studies concerning, the global emission of $\mathrm{CO}_{2}$ (see Gutiérrez et al. [8]).

\section{The model and its basic probabilistic characteristics}

\subsection{The proposed model}

The stochastic model proposed is based on a homogeneous stochastic Rayleigh diffusion process (HSRDP), which is defined as a Markov process $\left\{X_{t} ; t \in[0, T]\right\}$, with values in $] 0, \infty[$, and sample paths that are almost surely continuous and with infinitesimal moments (drift and diffusion coefficient) that are given by (see Giorno et al. [3])

$$
A_{1}(x)=\frac{a}{x}+b x, \quad A_{2}(x)=\sigma^{2}
$$

where $\sigma, a$ and $b(b \neq 0)$ are real parameters.

If $f(y, t \mid x, s)$ denotes the transition probability density function (TPDF) of this process, under certain conditions of regularity, which are satisfied by (1) and with the initial condition $\lim _{t \rightarrow s} f(y, t \mid x, s)=\delta(y-x)$, the above-mentioned TPDF satisfies the Kolmogorov (Fokker-Planck) forward equation

$$
\frac{\partial f(y, t \mid x, s)}{\partial t}=-\frac{\partial\left[A_{1}(y) f(y, t \mid x, s)\right]}{\partial y}+\frac{1}{2} \frac{\partial^{2}\left[A_{2}(y) f(y, t \mid x, s)\right]}{\partial y^{2}}
$$

Alternatively, under known conditions of regularity (see for example, Wong and Hajek [17]) satisfied by (1), the above process can be considered as the unique solution (with a probability of 1) of Ito's stochastic differential equation (SDE)

$$
\mathrm{d} X_{t}=\left(\frac{a}{X_{t}}+b X_{t}\right) \mathrm{d} t+\sigma \mathrm{d} W_{t}, \quad X_{0}=x_{0},
$$

where $W_{t}$ is a standard Wiener process.

The unique solution (a.s.) to the above Eq. (2) can be obtained (see Giorno et al. [3]) and can be expressed as follows

$$
f(x, t \mid y, s)=\frac{2 b y^{-\alpha} x^{\alpha+1} \mathrm{e}^{-\alpha b(t-s)}}{\sigma^{2}\left(\mathrm{e}^{2 b(t-s)}-1\right)} \exp \left(\frac{-b\left(x^{2}+y^{2} \mathrm{e}^{2 b(t-s)}\right)}{\sigma^{2}\left(\mathrm{e}^{2 b(t-s)}-1\right)}\right) I_{\alpha}\left(\frac{2 b x y \mathrm{e}^{b(t-s)}}{\sigma^{2}\left(\mathrm{e}^{2 b(t-s)}-1\right)}\right)
$$

for $a>-\sigma^{2} / 2$ and with the zero-flux condition. In this expression $I_{\alpha}$ denotes the modified Bessel function of the first kind and $\alpha=a / \sigma^{2}-1 / 2$.

\subsection{Moments of the model}

In Giorno et al. [3], certain probabilistic aspects of the HSRDP are ignored; nevertheless, these are of great interest in statistical modelling methodology and in the forecasting of real cases, for example in calculating moment functions and the statistical estimation of the process parameters. In the present paper, we establish in an explicit way the conditioned moment functions of order $r$ for HSRDP and, in particular, the trend (mean) functions, both conditioned and nonconditioned, all of which are expressed by means of Kummer functions. 
By definition, the $k$-order conditional moment of the model is given by:

$$
m_{k}(t \mid s)=\mathbf{E}\left(X_{t}^{k} \mid X_{s}=x_{s}\right)=\int_{0}^{\infty} x^{k} f\left(x, t \mid x_{s}, s\right) \mathrm{d} x
$$

To simplify the calculations involved in the above, let us denote: $\lambda=\lambda(s, t)=b / \sigma^{2}\left(\mathrm{e}^{2 b(t-s)}-1\right)$ and $\xi=\xi(s, t)=$ $\lambda x_{s} \mathrm{e}^{b(t-s)}$. Then, in terms of $\lambda$ and $\xi$, the conditional moments of the HRSDP are

$$
m_{k}(t \mid s)=2 \lambda^{\alpha+1} \xi^{-\alpha} \mathrm{e}^{-\xi^{2} / \lambda} \int_{0}^{\infty} x^{\alpha+k+1} \mathrm{e}^{-\lambda x^{2}} I_{\alpha}(2 \xi x) \mathrm{d} x
$$

With the change variable $z=x^{2}$, we obtain

$$
m_{k}(t \mid s)=\lambda^{\alpha+1} \xi^{-\alpha} \mathrm{e}^{-\xi^{2} / \lambda} \int_{0}^{\infty} z^{\alpha+k / 2} \mathrm{e}^{-\lambda z} I_{\alpha}(2 \xi \sqrt{z}) \mathrm{d} z
$$

then, by the relation (see ref. [5], p. 720:6.643): for $\operatorname{Re}(\mu+v+1 / 2)>0$

$$
\int_{0}^{\infty} z^{\mu-1 / 2} \mathrm{e}^{-\beta z} I_{2 v}(2 \gamma \sqrt{z}) \mathrm{d} y=\frac{\Gamma(\mu+v+1 / 2)}{\Gamma(2 v+1)} \gamma^{-1} \beta^{-\mu} \exp \left(\frac{\gamma^{2}}{2 \beta}\right) M_{-\mu, v}\left(\frac{\gamma^{2}}{\beta}\right)
$$

where $M_{.,}$is a Whittaker function, and as a result

$$
m_{k}(t \mid s)=\frac{\Gamma(\alpha+k / 2+1)}{\Gamma(\alpha+1)} \lambda^{(\alpha-k+1) / 2} \xi^{-\alpha-1} \mathrm{e}^{-\xi^{2} / 2 \lambda} M_{-(\alpha+k+1) / 2, \alpha / 2}\left(\frac{\xi^{2}}{\lambda}\right)
$$

by means of the related (see ref. [5], p. 1059:9.220) with the confluent hypergeometric function $\Phi$ (Kummer function):

$$
M_{v, \mu}(x)=x^{(\mu+1) / 2} \mathrm{e}^{-x / 2} \Phi(\mu-v+1 / 2,2 \mu+1, x)
$$

we find that

$$
m_{k}(t / s)=\frac{\Gamma(\alpha+k / 2+1)}{\Gamma(\alpha+1)} \lambda^{-k / 2} \mathrm{e}^{-\xi^{2} / \lambda} \Phi\left(\alpha+k / 2+1, \alpha+1, \frac{\xi^{2}}{\lambda}\right)
$$

Finally, by the Kummer transformation (see ref. [5], p. 1058:9.212) $\Phi(\beta, \gamma, z)=\mathrm{e}^{z} \Phi(\gamma-\beta, \gamma,-z)$, we deduce that the final form of the $k$-order conditional moment of the model is

$$
m_{k}(t \mid s)=\frac{\Gamma(\alpha+k / 2+1)}{\Gamma(\alpha+1)} \lambda^{-k / 2} \Phi\left(-\frac{k}{2}, \alpha+1,-\frac{\xi^{2}}{\lambda}\right)
$$

From this expression, we deduce the conditional trend function of the process, which is expressed as

$$
\mathbf{E}\left(X_{t} \mid X_{s}=x_{s}\right)=\frac{\Gamma(\alpha+3 / 2)}{\Gamma(\alpha+1)}\left(\frac{b}{\sigma^{2}\left(\mathrm{e}^{2 b(t-s)}-1\right)}\right)^{-1 / 2} \times \Phi\left(-\frac{1}{2}, \alpha+1, \frac{-b x_{s}^{2}}{\sigma^{2}\left(1-\mathrm{e}^{-2 b(t-s)}\right)}\right) .
$$
form

From Eq. (4) and under the initial distribution $P\left(X_{0}=x_{0}\right)=1$, the trend function of the process takes the following

$$
\mathbf{E}\left(X_{t}\right)=\frac{\Gamma(\alpha+3 / 2)}{\Gamma(\alpha+1)}\left(\frac{b}{\sigma^{2}\left(\mathrm{e}^{2 b t}-1\right)}\right)^{-1 / 2} \Phi\left(-\frac{1}{2}, \alpha+1, \frac{-b x_{0}^{2}}{\sigma^{2}\left(1-\mathrm{e}^{-2 b t}\right)}\right)
$$

The two expressions (4) and (5) play a fundamental role in constituting the estimated trend functions (see following Section), which enable us to fit and predict the future evolution of the stochastic variable in question.

\subsection{Stationary distribution of the process}

Let us now analyse the existence of the stationary distribution and obtain the explicit expression for its density. In general (see Nobile et al. [15]) the density function of stationary distribution, $S(x)$, in a diffusion can be expressed, 
under specific conditions that satisfy the HSRDP, as

$$
S(x)=\frac{c}{A_{2}(x)} \exp \left[2 \int_{z}^{x} \frac{A_{1}(y)}{A_{2}(y)} \mathrm{d} y\right]
$$

where $z$ is an arbitrary point in the interval $] 0,+\infty]$, and $c$ is a constant to be determined by the following normalization condition:

$$
c=\left[\int_{0}^{+\infty} \frac{1}{A_{2}(x)} \exp \left(2 \int_{z}^{x} \frac{A_{1}(y)}{A_{2}(y)} \mathrm{d} y\right) \mathrm{d} x\right]^{-1}
$$

By applying the above results, we can deduce that for $b<0$, the density function of the stationary distribution of the HSRDP exists, and takes the form

$$
S(x)=\frac{2\left(-b / \sigma^{2}\right)^{\alpha+1} x^{2 \alpha+1} \mathrm{e}^{b x^{2} / \sigma^{2}}}{\Gamma(\alpha+1)}
$$

It can be shown straightforwardly that the function $S$ is the density of the square of a random variable that has a Gamma distribution with parameters $\alpha+1$ and $-\sigma^{2} / b$.

Use can be made of this expression to calculate the asymptotic moment of order $k$, and thus we have for $b<0$

$$
\mathbf{E}\left[X^{k}(\infty)\right]=\int_{0}^{\infty} x^{k} S(x) \mathrm{d} x=\frac{\left(-b / \sigma^{2}\right)^{-k / 2} \Gamma(\alpha+k / 2+1)}{\Gamma(\alpha+1)}
$$

The asymptotic trend function of the process is, for $b<0$

$$
\mathbf{E}[X(\infty)]=\frac{\left(-b / \sigma^{2}\right)^{-1 / 2} \Gamma(\alpha+3 / 2)}{\Gamma(\alpha+1)}
$$

It can be seen that the limit of the trend function in Eq. (5) (when $t$ tends to $\infty$ ) coincides with the asymptotic trend function in Eq. (6).

\section{Estimation and approximation}

In this section we obtain estimators of the parameters and consider the computational questions that are encountered. For the drift parameters ( $a$ and $b$ ), we use the maximum likelihood method based on continuous sampling, and for the diffusion coefficient (the parameter $\sigma$ ) we obtain an approximate numerical estimation, based on the approximation of Ito's equation, that characterises the model between consecutive observations during an observed sample path of the process.

\subsection{Estimation of drift parameters}

The drift parameters $a$ and $b$ are estimated from an observed sample path of the HSRDP. The process is assumed to be observed in the interval $[0 ; T]$; then, the log-likelihood function under certain conditions that are satisfied by the HSRDP (see, for example Lipster and Shiryayev [14] for the general case) is given by

$$
\log (\mathbb{L})=\frac{1}{\sigma^{2}} \int_{0}^{T}\left(\frac{a}{X_{t}}+b X_{t}\right) \mathrm{d} X_{t}-\frac{1}{2 \sigma^{2}} \int_{0}^{T}\left(\frac{a}{X_{t}}+b X_{t}\right)^{2} \mathrm{~d} t
$$

By applying the Maximum Likelihood method (deriving with respect to the parameters and equalling to zero), and after various operations that need not be detailed, the estimators of the parameters are found to be:

$$
\hat{a}=\frac{\left(\int_{0}^{T} X_{t}^{2} \mathrm{~d} t\right)\left(\int_{0}^{T} \mathrm{~d} X_{t} / X_{t}\right)-T \int_{0}^{T} X_{t} \mathrm{~d} X_{t}}{\left(\int_{0}^{T} \mathrm{~d} t / X_{t}^{2}\right)\left(\int_{0}^{T} X_{t}^{2} \mathrm{~d} t\right)-T^{2}}
$$




$$
\hat{b}=\frac{\left(\int_{0}^{T} \mathrm{~d} t / X_{t}^{2}\right)\left(\int_{0}^{T} X_{t} \mathrm{~d} X_{t}\right)-T\left(\int_{0}^{T} \mathrm{~d} X_{t} / X_{t}\right)}{\left(\int_{0}^{T} \mathrm{~d} t / X_{t}^{2}\right)\left(\int_{0}^{T} X_{t}^{2} \mathrm{~d} t\right)-T^{2}}
$$

By means of Ito's formula, the stochastic integrals present in the latter expressions can be transformed into RiemannStieljes integrals, and we then have

$$
\int_{0}^{T} \frac{\mathrm{d} X_{t}}{X_{t}}=\log \left(X_{T} / x_{0}\right)+\frac{\sigma^{2}}{2} \int_{0}^{T} \frac{\mathrm{d} t}{X_{t}^{2}} \quad \text { and } \quad \int_{0}^{T} X_{t} \mathrm{~d} X_{t}=\frac{X_{T}^{2}-x_{0}^{2}}{2}-\frac{\sigma^{2} T}{2}
$$

Therefore, the expressions of the maximum likelihood estimators can be expressed as follows:

$$
\begin{aligned}
& \hat{a}=\frac{\left(\int_{0}^{T} X_{t}^{2} \mathrm{~d} t\right)\left(\log \left(X_{T} / x_{0}\right)+\sigma^{2} / 2 \int_{0}^{T} \mathrm{~d} t / X_{t}^{2}\right)-T / 2\left(X_{T}^{2}-x_{0}^{2}-\sigma^{2} T\right)}{\left(\int_{0}^{T} \mathrm{~d} t / X_{t}^{2}\right)\left(\int_{0}^{T} X_{t}^{2} d t\right)-T^{2}} \\
& \hat{b}=\frac{1 / 2\left(\int_{0}^{T} \mathrm{~d} t / X_{t}^{2}\right)\left(X_{T}^{2}-x_{0}^{2}-\sigma^{2} T\right)-T\left(\log \left(X_{T} / x_{0}\right)+\sigma^{2} / 2 \int_{0}^{T} \mathrm{~d} t / X_{t}^{2}\right)}{\left(\int_{0}^{T} \mathrm{~d} t / X_{t}^{2}\right)\left(\int_{0}^{T} X_{t}^{2} \mathrm{~d} t\right)-T^{2}}
\end{aligned}
$$

In order to use the above expressions to estimate the parameters, we must have continuous observations. In practice, continuous sample paths are not usually observed. Rather, the state of the diffusion process is observed at a finite number of time instances $\left(0=t_{0}<t_{1}<\cdots<t_{n}=T\right)$. By the Markov properties, the likelihood function corresponding to such data is the product of transition densities (in the present case, has a complicate form) and it is very difficult to find the estimators explicitly. An alternative estimation procedure that is frequently utilised (see, for example refs. [4,9]) for such data is to use the continuous time maximum likelihood estimators with suitable approximations of the integrals that appear in the expressions (7) and (8); specifically, the Riemann-Stieljes integrals are approximated by means of the trapezoidal formula.

\subsection{Approximate estimator of the diffusion coefficient}

Different procedures have been used to approximate the estimator of the diffusion coefficient of SDEs in general. Examples of such procedures include [6] who used spline cubic [4,9], with the extension of the procedure proposed in ref. [1], and which is utilised below to obtain an approximate estimator of the $\sigma$ parameter of the HSRDP model being studied. The model we propose is as follows: by applying Ito's formula, we have

$$
\mathrm{d}\left(\frac{1}{X_{t}}\right)=-\frac{\mathrm{d} X_{t}}{X_{t}^{2}}+\frac{\sigma^{2} \mathrm{~d} t}{X_{t}^{3}} .
$$

The differentials shown in (9) can be approximated by consecutive observations of a sample paths of the process in $t-1$ and $t$, as follows:

$$
\mathrm{d}\left(\frac{1}{X_{t}}\right) \simeq \frac{1}{X_{t}}-\frac{1}{X_{t-1}} \quad \text { and } \quad \mathrm{d}\left(X_{t}\right) \simeq X_{t}-X_{t-1}
$$

By substituting these approximations in Eq. (9), an approximate estimator of the $\sigma$ parameter between the latter observations is found to be

$$
\hat{\sigma}_{(t-1, t)}=\left|X_{t}-X_{t-1}\right| \sqrt{X_{t} / X_{t-1}}
$$

For $n+1$ observations of a sample paths of the process, the resulting approximate estimator has the following expression:

$$
\hat{\sigma}=\frac{1}{n} \sum_{t=1}^{n}\left|X_{t}-X_{t-1}\right| \sqrt{X_{t} / X_{t-1}}
$$




\section{Application and simulation}

\subsection{Application}

The methodology presented in this paper was applied to study the data available on the evolution of the production of thermal electricity in countries in the Maghreb region, during the period 1980-2002. These data are expressed in billions of Kw-hours and may be consulted at http://www.eia.doe.gov/pub/international/iealf.

In the present study, we analyse the trend of the above-mentioned variable by fitting the observed data to the estimated trend function (ETF) and the estimated conditional trend function (ECTF) of HSRDP. These estimated functions are obtained by using Zehna's theorem, replacing the parameters with the expressions given in expressions (7), (8) and (10) in the expressions of the trend functions (4) and (5). The methodology can be summarised in the following two phases:

- Step 1: Use the first 22 data in the series of observations considered to estimate the parameters of the model, using expressions (7), (8) and (10). In addition, obtain the corresponding ETF and ECTF values for the given sub-series and obtain the fit for the variable being analysed by means of these two trend functions.

- Step 2: For the year 2002, predict the corresponding values for "production of thermal electricity" using the ETF and the ECTF, and compare the results with the corresponding observed data for the same year.

A Mathematical program was implemented to carry out the calculations required for the computational methodology proposed in Section 3. The values of the corresponding estimators are: $\hat{a}=64.41126, \hat{b}=0.01627$ and $\hat{\sigma}^{2}=2.56820$.

Table 1 summarizes the fit and prediction results, i.e. the observed data, and the values predicted by ETF and ECTF respectively, and Fig. 1 shows the fits and the predictions made using the ETF and the ECTF, respectively.

Table 1

Observed values and predicted values

\begin{tabular}{|c|c|c|c|}
\hline Years & Data & ETF & ECTF \\
\hline \multicolumn{4}{|c|}{ Observed values } \\
\hline 1980 & 16.99 & 16.99 & 16.99 \\
\hline 1981 & 19.71 & 20.741 & 20.741 \\
\hline 1982 & 22.1 & 24.032 & 23.089 \\
\hline 1983 & 25.34 & 27.022 & 25.223 \\
\hline 1984 & 28.49 & 29.802 & 28.193 \\
\hline 1985 & 32.01 & 32.427 & 31.143 \\
\hline 1986 & 35.0 & 34.934 & 34.494 \\
\hline 1987 & 38.25 & 37.348 & 37.373 \\
\hline 1988 & 41.06 & 39.688 & 40.529 \\
\hline 1989 & 41.53 & 41.969 & 43.276 \\
\hline 1990 & 43.87 & 44.203 & 43.737 \\
\hline 1991 & 44.72 & 46.398 & 46.036 \\
\hline 1992 & 46.91 & 48.561 & 46.874 \\
\hline 1993 & 48.67 & 50.700 & 49.035 \\
\hline 1994 & 51.06 & 52.81 & 50.776 \\
\hline 1995 & 53.11 & 54.921 & 53.145 \\
\hline 1996 & 54.01 & 57.012 & 55.182 \\
\hline 1997 & 56.53 & 59.095 & 56.077 \\
\hline 1998 & 59.68 & 61.172 & 58.587 \\
\hline 1999 & 63.22 & 63.246 & 61.730 \\
\hline 2000 & 65.4 & 65.320 & 65.269 \\
\hline 2001 & 68.08 & 67.396 & 67.451 \\
\hline \multicolumn{4}{|c|}{ Predicted values } \\
\hline 2002 & 70.26 & 69.475 & 70.137 \\
\hline
\end{tabular}



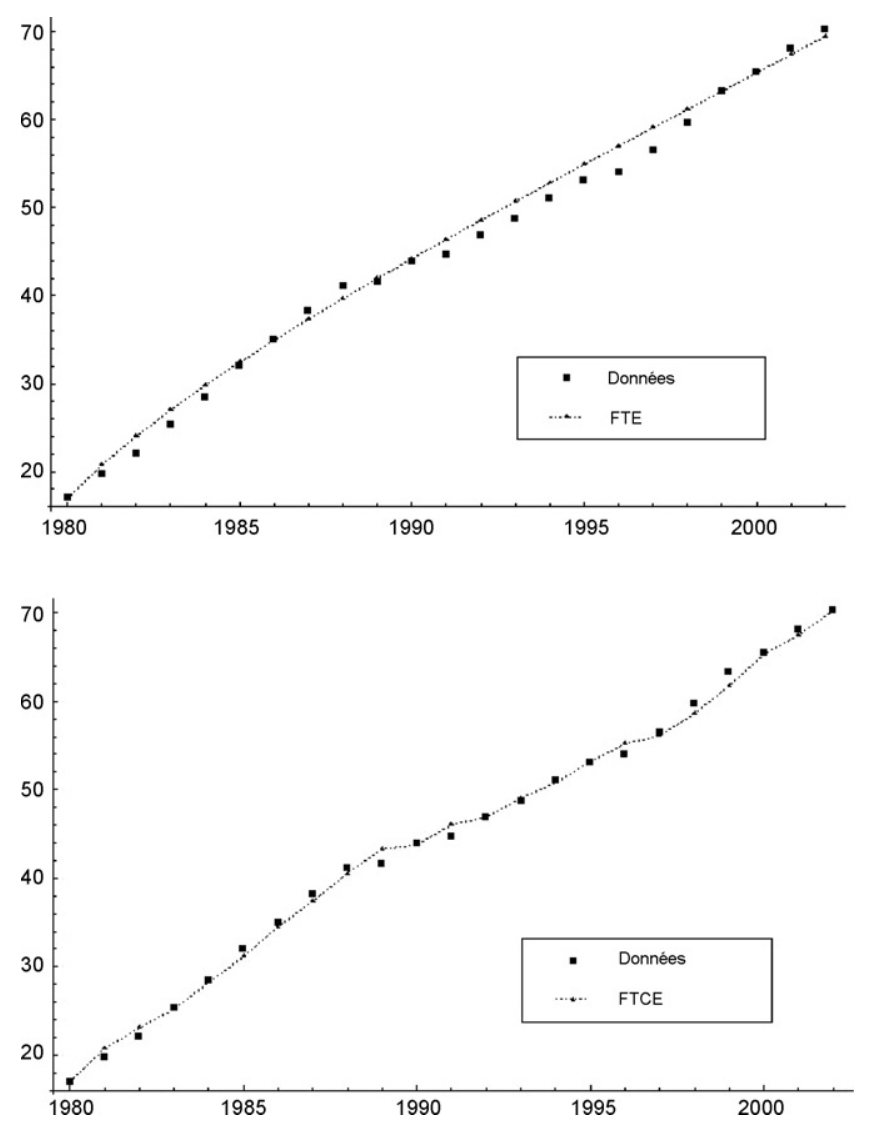

Fig. 1. Fit and forecast using ETF and ECTF.

\subsection{Simulation}

Given the form of the SDE (3), it is not possible to obtain the explicit expression of its solution. By using known general algorithms to simulate the SDEs, such as Taylor's algorithm to the order of 1.5 (see Kloeden and Platen [13] p. 351), an approximate solution can be found, from which we have

$$
x_{n+1}=x_{n}+h\left(\frac{a}{x_{n}}+b x_{n}\right)+\sigma \Delta W+\left(b-\frac{a}{x_{n}^{2}}\right) \sigma \Delta Z+\frac{h^{2}}{2}\left(b^{2} x_{n}-\frac{a^{2}-\sigma^{2} a}{x_{n}^{3}}\right)
$$

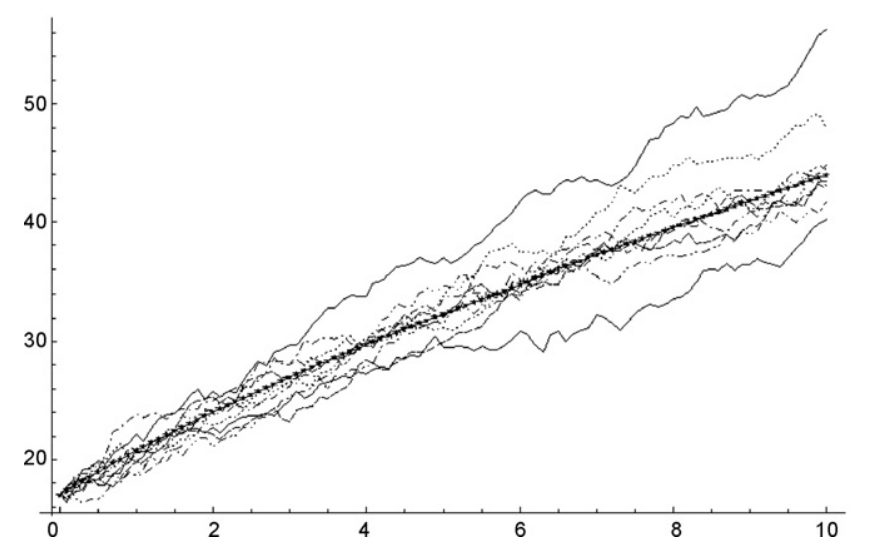

Fig. 2. A simulation of path. 
where $\Delta W=\sqrt{h} U_{1}$ and $\Delta Z=h^{3 / 2} / 2\left(U_{1}+U_{2} / \sqrt{3}\right)$, where $\Delta W=\sqrt{h} U_{1}$ and $\Delta Z=h^{3 / 2} / 2\left(U_{1}+U_{2} / \sqrt{3}\right)$, with $U_{1}$ and $U_{2}$ being two standard normal independent random variables, and where $h$ is the discretization step.

Fig. 2 illustrates the response pattern of ten sample paths with respect to their corresponding trend functions. In this simulation, we assumed $h=0.1$ and for the values of the parameters, we used those of their estimators that were obtained in the application.

In addition, a larger number $(n=100)$ of simulated trajectories have been obtained, which are not shown in the Fig. 2. The corresponding mean trajectory of this simulation tends significantly to fit the estimated trend function shown in Fig. 2. We do not yet possess measures of the accuracy of this fit, which will require a nonparametric methodology to deal with the question in an adequate way.

\section{Conclusion and discussion}

In this study, we tested the suitability of the HSRDP model for modelling real phenomena in the fields of energy and the environment. The possibilities of applying the HSRDP method considered here in other scientific fields have been explored in other recent studies, and notably in Demography (see ref. [7]). Moreover, we compared the practical possibilities of the estimation methodology proposed in Section 3. From the results obtained (Table 1 and Fig. 1), we conclude that the modelling of the real case considered, using the HSRDP model and the estimation methodology described in Section 3, provides a fit and a prediction, based on ETF and ECTF, that present a high degree of accuracy.

Another approach that was considered was to fit the case described in this paper using other diffusion models, such as those based on lognormal or on Gompertz homogeneous stochastic diffusions (see for example refs. [2,11,9]). For this purpose, a statistical-fit methodology was applied, but the fits achieved were unsatisfactory.

In the future, we shall address the (technically very complex) problem of obtaining results for a test of goodness of fit, at least in the asymptotic case, to enable us to establish a measure of the degree of accuracy in the fit and in the prediction and measures of estimation error. We shall also study the possibility of defining a nonhomogeneous Rayleigh model, introducing exogenous factors into the drift, in a similar way to what has already been achieved for other diffusions (see Gutiérrez et al. [11,10]). The authors are also currently investigating a statistical methodology for HSRDP based on estimation with discrete sampling, as an alternative to the continuous sampling that is considered in this paper.

\section{Acknowledgements}

The authors are very grateful to the editors and referees for constructive comments and suggestions. This work was supported partially by research project MTM 2005-09209, Ministerio de Educación y Ciencia, Spain.

\section{References}

[1] M. Chesney, J. Elliot, Estimating the instantaneous volatility and covariance of risky assets, Appl. Stochastic Models Data Anal. 11 (1995) 51-58.

[2] L. Ferrante, S. Bompade, L. Possati, L. Leone, Parameter estimation in a Gompertzian stochastic model for tumor growth, Biometrics 56 (2000) $1076-1081$.

[3] V. Giorno, A. Nobile, L.M. Ricciardi, L. Sacerdote, Some remarks on the Rayleigh process, J. Appl. Prob. 23 (1986) $398-408$.

[4] A.N. Giovanis, C.H. Skiadas, A stochastic logistic innovation diffusion-model studying the electricity consumption in Greece and the United States, Technol. Forecast. Soc. Change. 61 (1999) 253-264.

[5] L. Gradshteyn, I. Ryzhik, Table of Integrals, Series and Products, Academic Press, 1979.

[6] M. Guerra, L. Stefanini, A comparative simulation study for estimating diffusion coefficient, Math. Comput. Simul. 53 (2000) $193-203$.

[7] R. Gutiérrez, R. Gutiérrez-Sánchez, A. Nafidi, The Stochastic Rayleigh diffusion model: statistical inference and computational aspects. Applications to modelling of real cases, Appl. Math. Comput. 175 (2006) 628-644.

[8] R. Gutiérrez, R. Gutiérrez-Sánchez, A. Nafidi, E. Ramos, A diffusion model with cubic drift: statistical and computational aspects and application to modelling the global $\mathrm{CO}_{2}$ emission in Spain, Environmetrics 18 (2007) 55-69.

[9] R. Gutiérrez, A. Nafidi, R. Gutiérrez-Sánchez, Forecasting total natural-gas consumption in Spain by using the stochastic Gompertz innovation diffusion model, Appl. Energy 80 (2) (2005) 115-124.

[10] R. Gutiérrez, A. Nafidi, R. Gutiérrez-Sánchez, P. Román, F. Torres, Inference in Gompertz type nonhomogeneous stochastic systems by means of discrete sampling, Cybern. Syst. 36 (2005) 203-216.

[11] R. Gutiérrez, P. Román, F. Torres, Inference and first-passage-time for the lognormal diffusion process with exogenous factors: application to modelling in economics, Appl. Stochastic Models Business Ind. 15 (4) (1999) 325-332. 
[12] R. Gutiérrez, P. Román, F. Torres, Inference on some parametric functions in the univariate lognormal diffusion process with exogenous factors, Test 10 (2) (2001) 357-373.

[13] P. Kloeden, E. Platen, The Numerical Solution of Stochastic Differential Equations, Springer, Berlin, Germany, 1992.

[14] R.S. Lipster, A.N. Shiryayev, Statistics of Random Processes, Springer-Verlag, New York, 1981.

[15] A.G. Nobile, L.M. Ricciardi, Growth with regulation in fluctuating enviroments, Biol. Cybern. 49 (1984) 179-188.

[16] B. Prakasa Rao, Statistical Inference for Diffusion Type Process, Arnold, New York, 1999.

[17] E. Wong, B. Hajek, Stochastic Processes in Engineering Systems, Springer-Verlag, New York, 1985. 